\title{
On Some Properties of Pseudo-Semiregular Graphs
}

\section{Tamás Réti}

Bánki Donát Faculty of Mechanical and Safety Engineering, Óbuda University Népszínház u. 8, H-1081 Budapest, Hungary

reti.tamas@bgk.uni-obuda.hu

\section{Imre Felde}

John von Neumann Faculty of Informatics, Óbuda University

Bécsi út 96/b, H-1034 Budapest, Hungary

felde.imre@nik.uni-obuda.hu

Abstract: The application of the bipartite pseudo-semiregular graphs (BPS graphs) has recently had a growing importance in mathematical chemistry, mainly for QSAR (quantitative structure-activity relations) and QSPR (quantitative structure-property relations) studies. The aim of the research presented herein, is to give a systematic survey on the fundamental characteristics of BPS graphs and summarize some novel results concerning their structural-topological properties. We propose some practical methods for the construction of BPS graphs from various parent graphs. Moreover, a simple procedure is outlined by which a finite set of BPS graphs possessing the same spectral radius or the same second Zagreb index can be generated. Additionally, as a result of our investigations, it is verified that there is a strong correspondence between BPS graphs and balanced tree graphs of diameter 4.

Keywords: mathematical chemistry; strongly balanced tree graphs; Zagreb indices

\section{Introduction}

We consider only simple connected graphs. For a graph $\mathrm{G}$ with $\mathrm{n}$ vertices and $\mathrm{m}$ edges, $\mathrm{V}(\mathrm{G})$ and $\mathrm{E}(\mathrm{G})$ denote the set of vertices and edges, respectively. In this work our graph theoretical notation is standard and taken from [1].

An edge of $G$ connecting vertices $u$ and $v$ is denoted by uv. The degree $d(v)$ of a vertex $\mathrm{v}$ is the number of edges incident to $\mathrm{v}$. The finite set of vertex degrees of a graph $\mathrm{G}$ is denoted by $\mathrm{DS}(\mathrm{G})$. As usual, the cyclomatic number of a connected 
graph with $n$ vertices and $m$ edges is defined as $\mathrm{Cy}(\mathrm{G})=\mathrm{m}-\mathrm{n}+1$. A connected graph $\mathrm{G}$ having $\mathrm{Cy}(\mathrm{G})=\mathrm{k} \geq 1$ cycles is said to be a k-cyclic graph. As a particular case, if $\mathrm{k}=0$, the corresponding acyclic graph is called a tree graph. A tree with $\mathrm{n}$ vertices has exactly $n-1$ edges.

We denote by $\Delta=\Delta(\mathrm{G})$ and $\delta=\delta(\mathrm{G})$ the maximum and the minimum degrees of vertices of $\mathrm{G}$, and by $\mathrm{m}_{\mathrm{r}, \mathrm{s}}$ the total number of edges in $\mathrm{G}$ with end-vertex degrees $\mathrm{r}$ and $\mathrm{s}$, where we do not distinguish $\mathrm{m}_{\mathrm{r}, \mathrm{s}}$ and $\mathrm{m}_{\mathrm{s}, \mathrm{r}}$. For two different vertices $\mathrm{u}$ and $\mathrm{w}$, the distance $\mathrm{d}(\mathrm{u}, \mathrm{w})$ between $\mathrm{u}$ and $\mathrm{w}$ is the number of edges in a shortest path connecting them. The diameter of a connected graph $\mathrm{G}$ denoted by $\operatorname{diam}(\mathrm{G})$ is the maximum distance between any two vertices of $\mathrm{G}$.

Using the standard terminology [1], the path, cycle and star with $\mathrm{n}$ vertices are denoted by $P_{n}, C_{n}$ and $K_{1, n-1}$, respectively. Let $A=A(G)$ be the adjacency matrix of $\mathrm{G}$. The set of eigenvalues of $\mathrm{A}(\mathrm{G})$ is the spectrum of graph $\mathrm{G}$, and the largest eigenvalue of $A(G)$ denoted by $\rho(G)$ is called the spectral radius of $G$.

An integral graph is a graph with integral eigenvalues. A graph is $R$-regular if all its vertices have the same degree $\mathrm{R}$. A connected graph $\mathrm{G}$ is said to be bidegreed with degrees $\Delta$ and $\delta$ if at least one vertex of $\mathrm{G}$ has degree $\Delta$ and at least one vertex has degree $\delta$, and if no vertex of $\mathrm{G}$ has a degree different from $\Delta$ or $\delta$.

A connected bidegreed bipartite graph is called semiregular if each vertex in the same part of bipartition has the same degree. A connected graph $G$ is said to be harmonic (pseudo-regular) $[2,3,4]$ if there exists a positive constant $\mathrm{p}(\mathrm{G})$ such that each vertex $u$ of $G$ has the same average neighbor degree number $[2,3]$ identical with $p(G)$. The spectral radius of a harmonic graph $G$ is equal to $p(G)$. It is obvious [2] that any connected R-regular graph $G_{R}$ is a harmonic graph with $p\left(G_{R}\right)=\rho\left(G_{R}\right)=R$. A connected bipartite graph is called pseudo-semiregular $[2,5]$ if each vertex in the same part of bipartition has the same average degree. It is known among bipartite pseudo-semiregular graphs (BPS graphs) there exist connected graphs which are harmonic and pseudo-semiregular simultaneously.

By definition, a tree graph is called a balanced tree, if it has precisely one center vertex $\mathrm{u}$ for which $\Delta \geq \mathrm{d}(\mathrm{u}) \geq 2$ holds, and their vertices at the same distance $\mathrm{j}$ (at $\mathrm{j}^{\text {th }}$ level with $\mathrm{j}=1,2, . ., \mathrm{k}$ ) from the center have the same degree [6]. The simplest types of balanced trees are the $\mathrm{n}$-vertex stars $\mathrm{K}_{1, \mathrm{n}-1}$ having diameter 2 .

Define the first and second Zagreb indices $\left(\mathrm{M}_{1}\right.$ and $\left.\mathrm{M}_{2}\right)$ of a graph $\mathrm{G}$ as usual:

$$
\mathrm{M}_{1}=\mathrm{M}_{1}(\mathrm{G})=\sum_{\mathrm{u} \in \mathrm{V}} \mathrm{d}^{2}(\mathrm{u}) \quad \mathrm{M}_{2}=\mathrm{M}_{2}(\mathrm{G})=\sum_{\mathrm{uv} \in \mathrm{E}} \mathrm{d}(\mathrm{u}) \mathrm{d}(\mathrm{v}) .
$$

The Zagreb indices belong to the family of global topological graph invariants which are widely used in the mathematical chemistry. For more detailed information on Zagreb indices, we refer the reader to surveys [7-16]. A connected 
graph $G$ for which identity $\rho(G)=\sqrt{M_{2}(G) / m}$ holds is called a $Z_{2}$ graph, because it is defined on the basis of the second Zagreb index [16].

The paper is organized as follows. In Section 2, the fundamental features of $\mathrm{Z}_{2}$ graphs are reported. In Section 3, we summarize old and new results demonstrating some relevant properties of BPS graphs. Practical procedures for generating cyclic BPS graphs are presented in Section 4, where we also outline a method for the construction of an infinite set of BPS graphs with an identical spectral radius and/or a second Zagreb index. In Section 5, starting with the discussion of the spectral properties of tree graphs, we introduce the term of strongly balanced trees characterized by diameter 4 , and we demonstrate that it is possible to construct infinitely many strongly balanced trees with integer spectral radii.

\section{Some Properties of $Z_{2}$ Graphs}

It is important to note that if $G$ is an m-edge $Z_{2}$ graph then $\rho^{2}(G)=M_{2}(G) / m$ is a positive integer. The harmonic and bipartite pseudo-semiregular graphs form a subset of $Z_{2}$ graphs [17]. Until now, the types of $Z_{2}$ graphs have not exactly been identified and characterized. All regular graphs are $\mathrm{Z}_{2}$ graphs.

Denote by $\mathbf{j}$ the $\mathrm{n}$-component all-one vector. It is known that all semi-harmonic graphs for which $\mathrm{A}^{3} \mathbf{j}=\rho^{2} \mathrm{~A} \mathbf{j}$ holds, belong to the family of $Z_{2}$ graphs $[16,17,18]$. Consequently, the regular, semiregular, harmonic and bipartite pseudosemiregular graphs are the subsets of semi-harmonic graphs $[16,18]$.

In Fig. 1 various types of $Z_{2}$ graphs with diameter 4 and integer spectral radius are depicted.

- Graph $G_{A}$ is a pseudo-semiregular, non-harmonic graph with $\rho\left(G_{A}\right)=3$, $\operatorname{DS}\left(\mathrm{G}_{\mathrm{A}}\right)=\{1,2,4,5\}$ and $\mathrm{Cy}\left(\mathrm{G}_{\mathrm{A}}\right)=2$

- Graph $G_{B}$ is a harmonic, non-pseudo-semiregular graph with $\rho\left(G_{B}\right)=4$, $\operatorname{DS}\left(\mathrm{G}_{\mathrm{B}}\right)=\{1,4,7\}, \mathrm{Cy}\left(\mathrm{G}_{\mathrm{B}}\right)=7[2]$

- Graph $G_{C}$ is a harmonic, pseudo-semiregular graph with $\rho\left(G_{C}\right)=3$, $\operatorname{DS}\left(\mathrm{G}_{\mathrm{C}}\right)=\{1,2,3,6\}, \mathrm{Cy}\left(\mathrm{G}_{\mathrm{C}}\right)=3[4]$

- Graph $\mathrm{G}_{\mathrm{D}}$ is a harmonic, bipartite pseudo-semiregular, and balanced tree graph with $\rho\left(\mathrm{G}_{\mathrm{D}}\right)=3, \mathrm{DS}\left(\mathrm{G}_{\mathrm{D}}\right)=\{1,3,7\}$ and $\mathrm{Cy}\left(\mathrm{G}_{\mathrm{D}}\right)=0$ [19] 

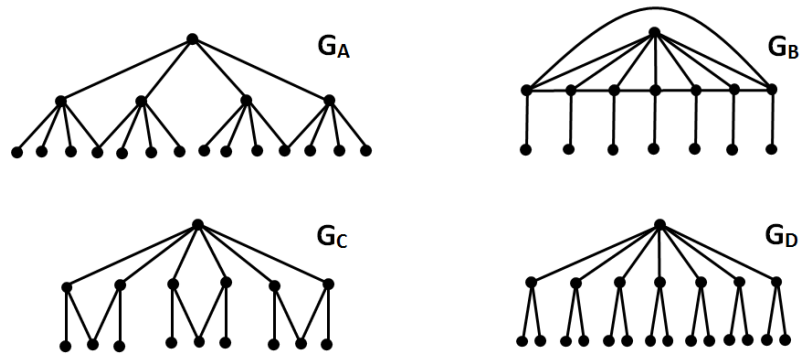

Figure 1

Four graphs with diameter 4 and integer spectral radius

However, there exist "sporadic" $Z_{2}$ graphs, not belonging to the graph families mentioned above. (They are neither harmonic nor bipartite pseudo-semiregular graphs.) The occurrence of such sporadic $\mathrm{Z}_{2}$ graphs is a mysterious phenomenon.

As an example, a bipartite and a non-bipartite sporadic graph belonging to set $Z_{2}$ are depicted in Fig. 2. For these graphs the corresponding spectral radii are: $\rho\left(J_{A}\right)=$ $\sqrt{5}$ and $\rho\left(J_{B}\right)=3$.
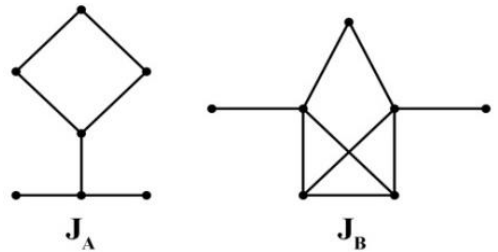

Figure 2

A bipartite graph $\left(\mathrm{J}_{\mathrm{A}}\right)$ and a non-bipartite graph $\left(\mathrm{J}_{\mathrm{B}}\right)$ belonging to set of $Z_{2}$ graphs

\section{On the Characterization of BPS Graphs}

The term of BPS graphs representing a subset of $Z_{2}$ graphs was introduced by $Y u$ et al. in 2004 (see Ref. [2]). Until now, only some elementary properties of $Z_{2}$ graphs and BPS graphs have been established, and for their structural characterization, except in very few studies [2, 5, 16, 17], only limited attempts have been made.

As we have already mentioned, a pseudo-semiregular graph $G$ is bipartite, consequently, the vertices of $G$ can be partitioned as $V=V_{1} U_{2}$. Denoted by $\mathrm{p}_{1}=\mathrm{p}_{1}\left(\mathrm{~V}_{1}\right)$ and $\mathrm{p}_{2}=\mathrm{p}_{2}\left(\mathrm{~V}_{2}\right)$ as the corresponding average degrees of vertices in $\mathrm{V}_{1}$ and $V_{2}$, respectively, the spectral radius of $G$ can be calculated as $\rho(G)=\sqrt{p_{1} p_{2}}$ [2]. Because for a semiregular graph the equality $\mathrm{p}_{1}\left(\mathrm{~V}_{1}\right)=\mathrm{p}_{2}\left(\mathrm{~V}_{2}\right)$ holds, this implies 
that any semiregular graph is a bipartite pseudo-semiregular graph. It is conjectured that the maximal cardinality of the degree sets of BPS graphs is not larger than 4. (See graphs $G_{A}$ and $G_{C}$ in Fig. 1.)

It is easy to construct planar acyclic BPS graphs with diameter 4 .

Proposition 1 For any $\mathrm{k} \geq 2$ integer there exist $\mathrm{k}$-cyclic BPS graphs with diameters not larger than 4 . The maximum vertex degree $\Delta$ of these cyclic graphs can be arbitrary large. This implies that their corresponding spectral radii can be arbitrary large, as well.

Proof. In Figs. 3-5 it is demonstrated that for any $\mathrm{k} \geq 2$ integer there exist k-cyclic pseudo-semiregular planar graphs composed only of quadrilaterals.

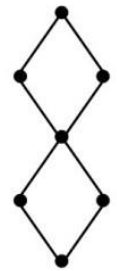

$\mathrm{B}_{2}$

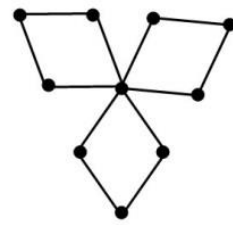

$\mathrm{B}_{3}$

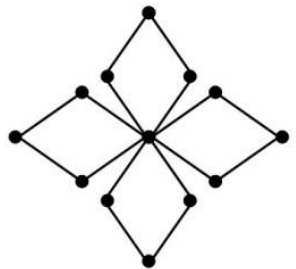

$\mathrm{B}_{4}$

Figure 3

Bidegreed cyclic pseudo-semiregular graphs $B_{k}$ including $k=2,3,4$ cycles

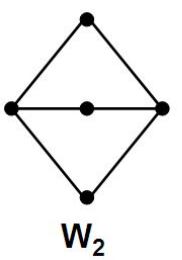

$\mathrm{W}_{2}$

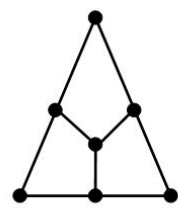

$\mathbf{W}_{3}$

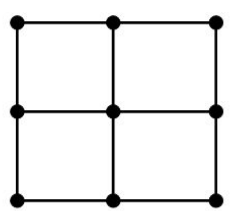

$\mathrm{W}_{4}$

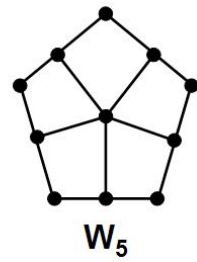

$\mathrm{W}_{5}$

Figure 4

Cyclic pseudo-semiregular graphs $\mathrm{W}_{\mathrm{k}}$ including $\mathrm{k}=2,3,4,5$ cycles

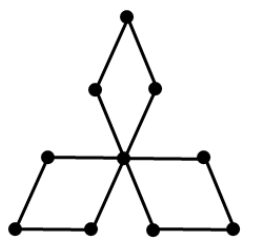

$\mathrm{H}_{3}$

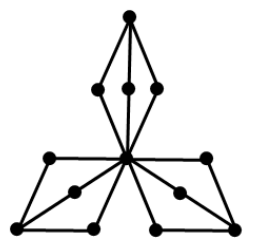

$\mathrm{H}_{6}$

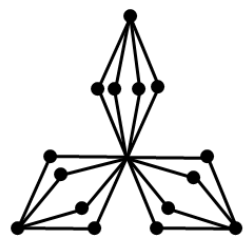

$\mathrm{H}_{9}$

Figure 5

Cyclic pseudo-semiregular graphs $\mathrm{H}_{\mathrm{k}}$ including $\mathrm{k}=3,6$ and 9 cycles 
A common property of planar BPS graphs $B_{k}, W_{k}$ and $H_{k}$, depicted in Figs. 3-5, is that they include $\mathrm{k}$ quadrilaterals. Graphs $\mathrm{W}_{2}$ and $\mathrm{W}_{3}$ are characterized by diameter 2 and 3, respectively, while the others have diameter 4 . Moreover, it is easy to show that depending on the appropriate choice of parameter $\mathrm{k}$ we can construct graphs BPS, with integer spectral radii, for example, $\rho\left(B_{7}\right)=4, \rho\left(W_{5}\right)=3$ and $\rho\left(\mathrm{H}_{9}\right)=4$.

It is worth noting that there exist BPS graphs characterized by extremal relations between the spectral radius and graph diameter. It is easy to show that path $\mathrm{P}_{5}$ is a BPS graph, and among all connected graphs with vertex number 5, the path $\mathrm{P}_{5}$ has the minimal spectral radius. Moreover, it has been shown in [20] that among all connected graphs on 11 vertices and diameter 4, the bicyclic BPS graph denoted by $\mathrm{Q}_{4,4,4}$ has the minimal spectral radius $\rho\left(\mathrm{Q}_{4,4,4}\right)=2.236068$. The bidegreed planar BPS graph $\mathrm{Q}_{4,4,4}$ is depicted in Fig. 6.

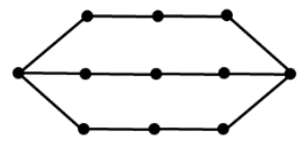

Figure 6

Extremal graph $\mathrm{Q}_{4,4,4}$ with minimal spectral radius

\section{Construction of BPS Graphs}

A general method to construct BPS graphs is based on the use of edge-subdivision operations performed on connected graphs. By definition, an edge-subdivision of a graph $\mathrm{G}$ results from inserting a new vertex in every edge of $\mathrm{G}$. For a connected graph $\mathrm{G}$, the corresponding subdivision graph is denoted by $\mathrm{S}(\mathrm{G})$.

\subsection{Construction of BPS Graphs from Semi-regular Graphs}

Pseudo-semiregular graphs can be easily constructed from semiregular graphs by using a subdivision graph operation. Let $\mathrm{G}(\Delta, \delta)$ be a semiregular graph. Performing a subdivision on $\mathrm{G}(\Delta, \delta)$ we get a pseudo-semiregular graph $\mathrm{S}(\mathrm{G}(\Delta, \delta))$ with spectral radius $\rho(\mathrm{S}(\mathrm{G}(\Delta, \delta)))=\sqrt{\Delta+\delta}$.

Example 1: Using an edge-subdivision operation on the star $\mathrm{K}_{1, \mathrm{n}-1}$, one obtains the tridegreed pseudo-semiregular tree $\mathrm{S}\left(\mathrm{K}_{1, \mathrm{n}-1}\right)$ with diameter 4 and spectral radius $\rho\left(\mathrm{S}\left(\mathrm{K}_{1, \mathrm{n}-1}\right)\right)=\sqrt{\Delta+1}=\sqrt{\mathrm{n}}$.

Example 2: Consider the semi-regular polyhedral graph $\mathrm{J}(4,3)$ with maximum degree 4 and minimum degree 3 . The bidegreed graph $J(4,3)$ with diameter 4 is the edge graph of the rhombic dodecahedron (See. Fig. 7). As a result of 
subdivision, from $\mathrm{J}(4,3)$ we obtain a tridegreed pseudo-semiregular graph $\mathrm{S}(\mathrm{J}(4,3))$ with diameter 8 . It is composed of octagons only and its spectral radius is $\rho(\mathrm{S}(\mathrm{J}(4,3)))=\sqrt{4+3}=\sqrt{7}$.

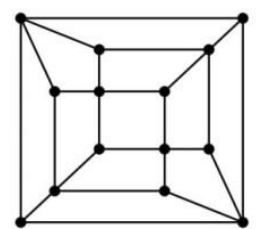

$\mathrm{J}(4,3)$

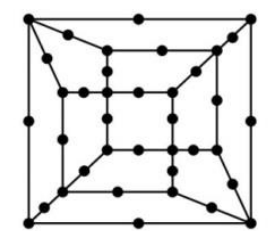

$\mathrm{S}(\mathrm{J}(4,3))$

Figure 7

Construction of a tridegreed pseudo-semiregular graph using a single subdividing operation on the rhombic dodecahedron graph

\subsection{Construction of BPS Graphs from Connected $R \geq 3$ Regular Graphs}

Let $G_{R}$ be an $R \geq 3$ regular connected graph. Performing on $G$ a double edgesubdividing we get the bidegreed pseudo-semiregular graph $\mathrm{S}\left(\mathrm{S}\left(\mathrm{G}_{\mathrm{R}}\right)\right)$. The spectral radius of resulting pseudo-semiregular graph will be $\rho\left(S\left(S\left(G_{R}\right)\right)\right)=\sqrt{R+2}$. This implies that the spectral radius of graph $\mathrm{S}\left(\mathrm{S}\left(\mathrm{G}_{\mathrm{R}}\right)\right)$ will be integer if $(2+\mathrm{R})$ is a perfect square.

\subsection{Construction of BPS Graphs with Identical Spectral Radii or Second Zagreb Indices}

For unicyclic pseudo-semiregular graphs the following proposition can be verified:

Proposition 2 There exist unicyclic BPS graphs with arbitrary large spectral radii and arbitrary large diameters.

Proof. Consider the thorn-like graph $\mathrm{G}_{\mathrm{t}, \mathrm{q}}$ depicted in Fig. 8. It is constructed from a cycle $\mathrm{C}_{2 q}$ of length $2 \mathrm{q}$ by attaching $\mathrm{t} \geq 1$ terminal vertices (pendant edges) to every second vertices of the cycle.

The number of pendant vertices is $n_{1}=t q$, the number of vertices with degree 2 is $\mathrm{n}_{2}=\mathrm{q}$, and the number of vertices of maximal degree is $\mathrm{n}_{\mathrm{t}+2}=\mathrm{q}$. This implies that the total number of vertices will be $n=q(2+t)$. It is easy to see that for the edge parameters we have: $\mathrm{m}_{1, \mathrm{t}+2}=\mathrm{qt}, \mathrm{m}_{2, \mathrm{t}+2}=2 \mathrm{q}$, consequently, $\mathrm{m}\left(\mathrm{G}_{\mathrm{t}, \mathrm{q}}\right)=\mathrm{n}\left(\mathrm{G}_{\mathrm{t}, \mathrm{q}}\right)=\mathrm{q}(2+\mathrm{t})$. 


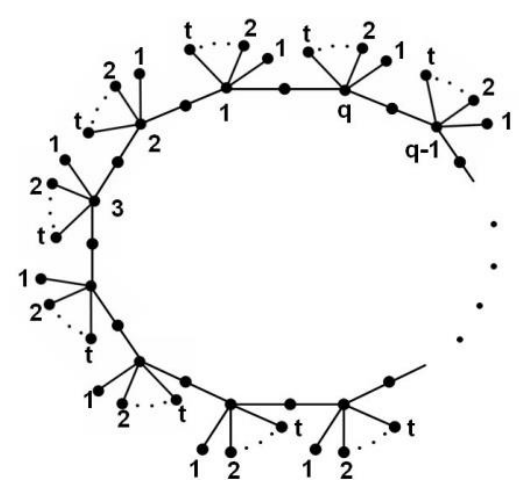

Figure 8

A tridegreed, unicyclic pseudo-semiregular graph, $\mathrm{G}_{\mathrm{t}, \mathrm{q}}$

Because the graph $\mathrm{G}_{\mathrm{t}, \mathrm{q}}$ is pseudo-semiregular, the corresponding first and the second Zagreb indices are: $\mathrm{M}_{1}\left(\mathrm{G}_{\mathrm{t}, \mathrm{q}}\right)=\mathrm{q}\left(\mathrm{t}^{2}+5 \mathrm{t}+8\right)$ and $\mathrm{M}_{2}\left(\mathrm{G}_{\mathrm{t}, \mathrm{q}}\right)=\mathrm{q}(\mathrm{t}+2)(\mathrm{t}+4)$, the maximum vertex degree $\Delta\left(\mathrm{G}_{\mathrm{t}, \mathrm{q}}\right)=\mathrm{t}+2$.

Moreover, $\mathrm{G}_{\mathrm{t}, \mathrm{q}}$ is a $\mathrm{Z}_{2}$ graph, this implies that its spectral radius is

$$
\rho\left(G_{t, q}\right)=\sqrt{\frac{M_{2}\left(G_{t, q}\right)}{m\left(G_{t, q}\right)}}=\sqrt{t+4}=\sqrt{\Delta+2}
$$

From the above results the following conclusions can be drawn:

(i) For any fixed parameter $\mathrm{q} \geq 2$, if $\mathrm{t}$ tends to infinity, then the maximum vertex degree $\Delta\left(\mathrm{G}_{\mathrm{t}, \mathrm{q}}\right)=\mathrm{t}+2$ and the spectral radius $\rho\left(\mathrm{G}_{\mathrm{t}, \mathrm{q}}\right)$ tend to infinity.

(ii) For a fixed parameter $\mathrm{t} \geq 1$, if $\mathrm{q}$ tends to infinity, then the diameter $\operatorname{diam}\left(\mathrm{G}_{\mathrm{t}, \mathrm{q}}\right)$ tends to infinity.

(iii) The spectral radius $\rho\left(G_{t, q}\right)$ depends only on the parameter $t$. If the sum $\mathrm{t}+4$ is a perfect square then the spectral radius will be a positive integer.

Based on the previous considerations, it is easy to construct non-isomorphic pseudo-semiregular graphs with identical spectral radius or second Zagreb index.

\subsection{Construction of an Infinite Set of $G_{t, q}$ Graphs with an Identical Spectral Radius}

A simple method which can be used for generating an infinite family of $G_{t, q}$ graphs with identical spectral radius is demonstrated in the following example: Let $\mathrm{t}=5$. In this case, for any graph $\mathrm{G}_{5, \mathrm{q}}$ the spectral radius will be the same integer, $\rho\left(G_{5, q}\right)=3$ for arbitrary $q \geq 2$. Then for given parameter pairs $(t=5, q \geq 2)$ the corresponding edge numbers and second Zagreb indices of graphs $\mathrm{G}_{5, \mathrm{q}}$ can be simply determined. The computed results are given in Table 1. 
Table 1

Computed topological parameters for graphs $\mathrm{G}_{\mathrm{t}, \mathrm{q}}$ having an identical spectral radius

\begin{tabular}{|c|c|c|c|c|c|c|c|}
\hline Graph & $\mathbf{t}$ & $\mathbf{q}$ & $\boldsymbol{\rho}$ & $\mathbf{m}=\mathbf{q}(\mathbf{2}+\mathbf{t})$ & $\mathbf{M}_{\mathbf{2}}=\mathbf{m} \boldsymbol{\rho}^{\mathbf{2}}$ & $\begin{array}{c}\mathbf{M}_{\mathbf{1}}=\mathbf{q}\left(\mathbf{t}^{\mathbf{2}}+\mathbf{5 t}+\right. \\
\mathbf{8})\end{array}$ & $\begin{array}{c}\mathbf{Z}=\mathbf{M}_{\mathbf{2}} / \mathbf{m}- \\
\left.\mathbf{M}_{\mathbf{1}} / \mathbf{n}=\mathbf{t} / \mathbf{( 2 + t}\right)\end{array}$ \\
\hline $\mathrm{G}_{5,2}$ & 5 & 2 & 3 & 14 & 126 & 116 & $5 / 7$ \\
\hline $\mathrm{G}_{5,3}$ & 5 & 3 & 3 & 21 & 189 & 174 & $5 / 7$ \\
\hline $\mathrm{G}_{5,4}$ & 5 & 4 & 3 & 28 & 252 & 232 & $5 / 7$ \\
\hline $\mathrm{G}_{5,5}$ & 5 & 5 & 3 & 35 & 315 & 290 & $5 / 7$ \\
\hline $\mathrm{G}_{5,6}$ & 5 & 6 & 3 & 42 & 378 & 348 & $5 / 7$ \\
\hline $\mathrm{G}_{5,7}$ & 5 & 7 & 3 & 49 & 441 & 406 & $5 / 7$ \\
\hline $\mathrm{G}_{5,8}$ & 5 & 8 & 3 & 56 & 504 & 464 & $5 / 7$ \\
\hline $\mathrm{G}_{5,9}$ & 5 & 9 & 3 & 63 & 567 & 522 & $5 / 7$ \\
\hline $\mathrm{G}_{5,10}$ & 5 & 10 & 3 & 70 & 630 & 580 & $5 / 7$ \\
\hline
\end{tabular}

Because graphs $\mathrm{G}_{\mathrm{t}, \mathrm{q}}$ are $\mathrm{Z}_{2}$ graphs, their spectral radius is equal to $\rho\left(G_{5, q}\right)=\sqrt{M_{2}\left(G_{5, q}\right) / m\left(G_{5, q}\right)}=3$ for any $q \geq 2$. Additionally, in the last column of

Table 1 , the quantity denoted by $\mathrm{Z}$ is given. Topological parameter $\mathrm{Z}=\mathrm{Z}(\mathrm{G})$ characterizes the structure of a graph $G$ [17]. It is interesting to note that for all graphs included in Table 1 the values of $\mathrm{Z}$ are identical.

\subsection{Construction of $\mathbf{G}_{\mathrm{t}, \mathrm{q}}$ Graphs with an Identical Second Zagreb Index}

The concept outlined previously, is applicable for generating $G_{t, q}$ graphs with an identical second Zagreb index. Let $\mathrm{t}=\mathrm{q} \geq 2$ be an arbitrary positive integer. As a first step, for graph $G_{t, t}$, we calculate its second Zagreb index given as $\mathrm{M}_{2}\left(\mathrm{G}_{\mathrm{t}, \mathrm{t}}\right)=\mathrm{t}(\mathrm{t}+2)(\mathrm{t}+4)$. As a second step, by using a computer search, we identify all graphs $\mathrm{G}_{\mathrm{t}, \mathrm{q}}$ which satisfy the equality represented by $\mathrm{m}=\mathrm{M}_{2}\left(\mathrm{G}_{\mathrm{t}, \mathrm{t}}\right) / \rho^{2}\left(\mathrm{G}_{\mathrm{t}, \mathrm{q}}\right)=\mathrm{q}(\mathrm{t}+2)$, where $\mathrm{t}$ and $\mathrm{q}$ are positive integers. It can be expected that there exists a finite set of graphs $\mathrm{G}_{\mathrm{t}, \mathrm{q}}$ with different $(\mathrm{t}, \mathrm{q})$ parameters for which the above equality holds. The method based on a simple computer search is demonstrated in the following example. Let $\mathrm{t}=\mathrm{q}=10$. In this case, $\mathrm{M}_{2}\left(\mathrm{G}_{10,10}\right)=1680$ and there are exactly 8 non-isomorphic $\mathrm{G}_{\mathrm{t}, \mathrm{q}}$ graphs satisfying the requirements of computer search. All of them have the same second Zagreb index 1680. The computed results are summarized in Table 2 .

As can be seen, among $\mathrm{G}_{\mathrm{t}, \mathrm{q}}$ graphs there exist graph pairs having equal first and second Zagreb indices as well. Namely, for such graphs the equalities $M_{1}\left(G_{1,112}\right)=$ $\mathrm{M}_{1}\left(\mathrm{G}_{8,14}\right)=1568$ and $\mathrm{M}_{1}\left(\mathrm{G}_{2,70}\right)=\mathrm{M}_{1}\left(\mathrm{G}_{4,35}\right)=1540$ are fulfilled. 
Table 2

Computed topological parameters for graphs $\mathrm{G}_{\mathrm{t}, \mathrm{q}}$ having identical second Zagreb index

\begin{tabular}{|c|c|c|c|c|c|c|c|}
\hline Graph & $\mathbf{t}$ & $\mathbf{q}$ & $\mathbf{m = q}(\mathbf{2 + t})$ & $\boldsymbol{\rho}^{\mathbf{2}}=\mathbf{4 + t}$ & $\mathbf{M}_{\mathbf{2}}=\mathbf{m} \boldsymbol{\rho}^{\mathbf{2}}$ & $\begin{array}{c}\mathbf{M}_{\mathbf{1}}=\mathbf{q}\left(\mathbf{t}^{\mathbf{2}} \mathbf{+ 5 t}\right. \\
\mathbf{+ 8})\end{array}$ & $\begin{array}{c}\mathbf{Z}=\mathbf{M}_{\mathbf{2}} / \mathbf{m}- \\
\mathbf{M}_{\mathbf{1}} \mathbf{n}\end{array}$ \\
\hline $\mathrm{G}_{1,112}$ & 1 & 112 & 336 & 5 & 1680 & $\underline{1568}$ & $1 / 3$ \\
\hline $\mathrm{G}_{2,70}$ & 2 & 70 & 280 & 6 & 1680 & $\underline{1540}$ & $1 / 2$ \\
\hline $\mathrm{G}_{3,48}$ & 3 & 48 & 240 & 7 & 1680 & 1536 & $3 / 5$ \\
\hline $\mathrm{G}_{4,35}$ & 4 & 35 & 210 & 8 & 1680 & $\underline{1540}$ & $2 / 3$ \\
\hline $\mathrm{G}_{6,21}$ & 6 & 21 & 168 & 10 & 1680 & 1554 & $3 / 4$ \\
\hline $\mathrm{G}_{8,14}$ & 8 & 14 & 140 & 12 & 1680 & $\underline{1568}$ & $4 / 5$ \\
\hline $\mathrm{G}_{10,10}$ & 10 & 10 & 120 & 14 & 1680 & 1580 & $5 / 6$ \\
\hline $\mathrm{G}_{26,2}$ & 26 & 2 & 56 & 30 & 1680 & 1628 & $13 / 14$ \\
\hline
\end{tabular}

\section{On the Spectral Properties of Tree Graphs}

In 1974, Harary and Schwenk initiated the problem of finding connected graphs having integral spectrum [21]. During the past few decades, for certain families of integral graphs, many novel results have been obtained [4, 6, 21-29]. For the construction of integral trees, some efficient methods have been developed [6, 22, 25-29]. Among others, it was proved [6] that integral balanced trees with diameter 5,7 and 9 do not exist, just like integral balanced trees with diameter $4 \mathrm{k}+1$ ( $\mathrm{k}$ is an arbitrary integer). Recently, it has been verified that there exist integral trees of arbitrary large diameters. Csikvári has constructed integral trees with arbitrary large even diameters [28], and Ghorbani et al. have settled the odd-diameter case [29]. It is worth noting that there exist integral trees with diameter 5 which belong to the family of $\mathrm{Z}_{2}$ graphs. The 25-vertex graph $\mathrm{T}_{25}$ depicted in Fig.9 is the smallest integral tree of diameter 5 with the spectrum $\left\{3,2^{3}, 1^{3}, 0^{11},-1^{3},-2^{3},-3\right\}$ [26]. Tree $T_{25}$ is a sporadic $Z_{2}$ graph because it does not belong to the sets of either harmonic or pseudo-semiregular graphs.

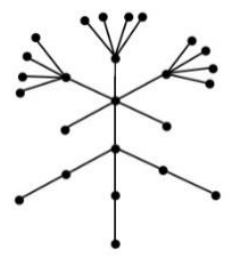

Figure 9

A "sporadic" integral tree belonging to the family of $Z_{2}$ graphs [26] 
Currently, the search for non-integral tree graphs characterized by integer spectral radius has gained raising interest $[30,31]$. Patuzzi et al. [31] investigated nonintegral trees, namely star-like trees and double brooms. For each class, they determined conditions for the spectral radius to be integer. Moreover, it was concluded that among trees of diameter 4 , there are infinitely many non-integral trees with integer spectral radius [30].

In the last decade, several authors have studied the spectral radius of connected graphs as a function of given graph invariants (fixed diameter $\operatorname{diam}(\mathrm{G})$ and largest vertex degree $\Delta$ ).

Van Dam [32] determined the graphs with maximal spectral radius among all graphs on $n$ vertices with $\operatorname{diam}(\mathrm{G}) \geq 2$. It is important to note that Hansen and Stevanović obtained the same result using a completely different approach [33]. This complements the results published in [20] on connected graphs with minimal spectral radius for a given number of vertices and diameter. Yuan et al. [34] determined the graphs having the minimal spectral radius among all the graphs on $n$ vertices with $\operatorname{diam}(G)=n-4$. The relevant results in this topic are summarized in [18]. Some of them will be used in our subsequent investigations.

Lovász and Pelikán [35] verified that among n-vertex trees the path $\mathrm{P}_{\mathrm{n}}$ has the minimal spectral radius and the star $\mathrm{K}_{1, \mathrm{n}-1}$ has the maximal spectral radius. In [36] Simić and Tošić identified the n-vertex trees $T_{n}, \Delta$, whose spectral radius has the maximal value among all trees with a fixed maximal degree $\Delta$. As an example, in Fig. 10 four trees are depicted. These 7-vertex trees are extremal graphs in a certain sense.

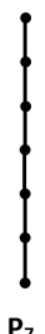

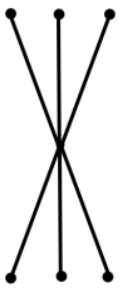

$\mathbf{K}_{1,6}$

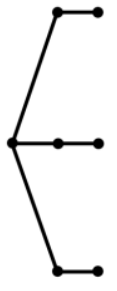

$T_{7, x}$

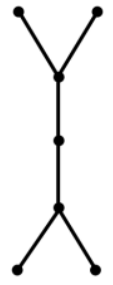

$T_{7, Y}$

Figure 10

Extremal 7-vertex trees having maximal or minimal spectral radius

Except path $\mathrm{P}_{7}$, all of them are BPS graphs. Path $\mathrm{P}_{7}$ is has the minimal spectral radius, and star $\mathrm{K}_{1,6}$ has the maximal spectral radius. Later on, it will be shown that $\mathrm{T}_{7, \mathrm{X}}$ and $\mathrm{T}_{7, \mathrm{Y}}$ represent the extremal trees which have identical minimal spectral radius among 7-vertex trees of diameter $4\left(\rho\left(T_{7, X}\right)=\rho\left(T_{7, Y}\right)=2\right)$. Moreover, their second Zagreb indices are also equal: $\mathrm{M}_{2}\left(\mathrm{~T}_{7, \mathrm{X}}\right)=\mathrm{M}_{2}\left(\mathrm{~T}_{7, \mathrm{Y}}\right)=24$.

Stevanović proved [37] that for the spectral radius of a tree $\mathrm{T}$ the general inequality $\rho(T) \leq 2 \sqrt{\Delta-1}$ holds where $\Delta$ is the maximal degree of $T$. In the most 
recent years the upper bound represented by the this inequality has been extensively studied and improved for some particular cases [18, 30, 38-42].

Chang and Huang [39] elaborated new ordering systems for n-vertex trees according to their spectral radius and diameter, and identified the tree types with maximal spectral radius. In [38] and [40] sharp upper bounds are given for the spectral radius of trees with diameter not larger than 4 .

Rojo and Robinho [41] studied the spectrum of Bethe trees. By definition, a Bethe tree denoted by $\mathrm{B}_{\Delta, \mathrm{k}}$ is a rooted tree of $\mathrm{k}$ levels in which the root vertex has a degree equal to $\Delta-1$, the vertices in level $\mathrm{j}(2 \leq \mathrm{j} \leq \mathrm{k}-1)$ have degree equal to $\Delta$ and the vertices in level $\mathrm{k}$ have degrees equal to 1 . They derived an explicit formula by which the nonzero eigenvalues of Bethe trees can be calculated [41]. The spectral radius $\rho\left(B_{\Delta, k}\right)$ of a Bethe tree of k levels is

$$
\rho\left(\mathrm{B}_{\Delta, \mathrm{k}}\right)=2 \sqrt{\Delta-1} \cos \left(\frac{\pi}{\mathrm{k}+1}\right)
$$

By introducing the term of the completely full-degree tree, Song et al. [38] obtained a new upper bound on the spectral radius of trees which are neither a path nor a star. By definition, an n-vertex balanced tree $\mathrm{T}_{\Delta, \mathrm{k}}$ with maximum degree $\Delta$ is called a completely full-degree tree if the degrees of all the vertices of $T_{\Delta, \mathrm{k}}$ are equal to $\Delta$, except the vertices in the last level $\mathrm{k}$, where each degree is equal to 1 . They verified [38] that if $\mathrm{T}$ is a $\mathrm{n}$-vertex tree with maximum degree $\Delta$, for which $2 \Delta<\mathrm{n} \leq \Delta^{2}+1$ is fulfilled, then $\rho(\mathrm{T}) \leq \sqrt{2 \Delta-1}$ with equality if and only if $\mathrm{T}$ is a completely full-degree tree of diameter 4 . As an example, a Bethe tree and a completely full-degree tree are shown in Fig. 11.

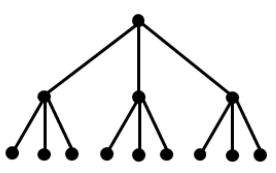

a

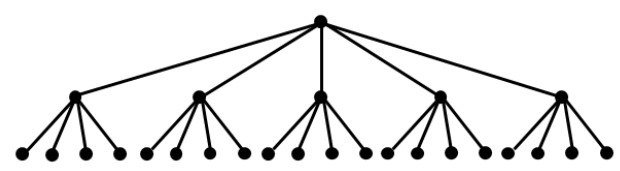

b

Figure 11

Bethe tree $\mathrm{B}_{4,3}$ (a) and the completely full-degree tree $\mathrm{T}_{5,3}$ with diameter four (b)

\subsection{On the Construction of Balanced Tree Graphs}

It is known that the diameter of a balanced tree having only one central vertex is even. Consequently, there are no balanced trees of diameter 3. The smallest balanced trees are the star $\mathrm{K}_{1,2}$ with diameter 2, and the path $\mathrm{P}_{5}$ with diameter 4 . Both of them are BPS graphs. 
Consider the Smith graphs $G(n)$ with $n \geq 7$ vertices [43]. (See Fig. 12.) A fundamental property of trees $\mathrm{G}(\mathrm{n})$ is that they have an identical integer spectral radius equal to 2 . If $n \geq 8$, the corresponding Smith graphs $G(n)$ represent an infinite class of sporadic $Z_{2}$ trees with arbitrary large diameter. If $n \geq 7$ is an odd integer, then graphs $G(n)$ are balanced trees. Moreover, it is easy to see that if $n=7$, then tree $G(7)$ is a balanced BPS graph which is isomorphic to tree $T_{7, Y}$ depicted in Fig. 10.

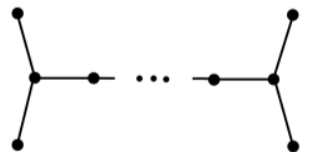

Figure 12

Smith graphs $\mathrm{G}(\mathrm{n})$ with $\mathrm{n} \geq 7$ vertices [43]

Using a subdivision operation on $\mathrm{G}(\mathrm{n})$ graphs we can generate novel balanced trees. If $n \geq 7$, then the corresponding subdivision graph $S(G(n))$ will be a balanced tree. The subdivision transformation can be repeated several times. Consequently, performing the subdivision transformation on $\mathrm{S}(\mathrm{G}(\mathrm{n}))$, we obtain the balanced tree $\mathrm{S}(\mathrm{S}(\mathrm{G}(\mathrm{n})))$. From this observation the following general conclusion can be drawn: The subdivision graph $S\left(T_{b}\right)$ of a balanced tree $T_{b}$ is a balanced tree.

The general topological structure of trees with diameter 4 is demonstrated in Fig. 13. As can be seen, their possible structure is determined by a large set of parameters denoted by $\left(b, r, a_{1}, a_{2}, \ldots, a_{r}\right)$.

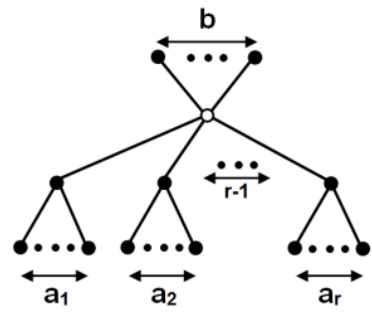

Figure 13

Tree $\mathrm{T}\left(\mathrm{b}, \mathrm{r}, \mathrm{a}_{1}, \mathrm{a}_{2}, \ldots, \mathrm{a}_{\mathrm{r}}\right)$ of diameter $4[30]$

There are some interesting types of n-vertex trees which form particular subsets of balanced trees with diameter 4 . Such graphs are the Bethe trees $B_{\Delta, 3}$ and completely full-degree trees $\mathrm{T}_{\Delta, 3}$. Patuzzi et al. [30] have proven that for the spectral radius of the tree $T\left(b, r, a_{1}, a_{2}, \ldots, a_{r}\right)$ with diameter 4 , the following sharp upper bound can be formulated:

$$
\rho\left(\mathrm{T}\left(\mathrm{b}, \mathrm{r}, \mathrm{a}_{1}, \mathrm{a}_{2}, \ldots, \mathrm{a}_{\mathrm{r}}\right) \leq \sqrt{2 \Delta-1}\right.
$$


In the above formula equality holds if and only if $b=0, a_{i}=a$ for $1 \leq \mathrm{i} \leq \mathrm{r}$ and $\Delta=\mathrm{a}+1=\mathrm{r}$.

It is easy to show that there is a strong correspondence between bipartite pseudosemiregular graphs and balanced tree graphs. In what follows we restrict our attention primarily to the relations between the BPS graphs and balanced tree graphs having diameter four.

\subsection{A New Class of Balanced BPS Trees with Diameter 4}

We define a particular class $\Omega_{4}$ of balanced trees with diameter 4 as follows: Let $d_{A} \geq 2$ and $d_{B} \geq 2$ be positive integers. By definition, a balanced tree $T\left(d_{A}, d_{B}\right)$ of 3 levels belongs to graph family $\Omega_{4}$ if the centrum vertex has degree $\mathrm{d}_{\mathrm{A}}$, the vertices at distance 1 from the centrum have degree $d_{B}$, and all vertices at distance 2 have degree 1. From the definition it follows that parameter pairs $\left(d_{A}, d_{B}\right)$ determine unambiguously the adjacency matrix of tree $T\left(d_{A}, d_{B}\right)$. It is easy to see that the edge number of $T\left(d_{A}, d_{B}\right)$ is equal to $m=m\left(T\left(d_{A}, d_{B}\right)\right)=d_{A} d_{B}$. For simplicity, graphs $\mathrm{T}\left(\mathrm{d}_{\mathrm{A}}, \mathrm{d}_{\mathrm{B}}\right)$ included in $\Omega_{4}$ are called strongly balanced trees.

Example 3 In a particular case, if $d_{A}=d_{B}=2$ holds, then the corresponding strongly balanced tree $\mathrm{T}(2,2)$ with $\mathrm{m}=4$ edges is isomorphic to the 5-vertex path $\mathrm{P}_{5}$ of diameter 4 .

Lemma 1 [15]: The strongly balanced tree $T\left(d_{A}, d_{B}\right)$ is a BPS graph.

Proof. The vertex set of the bipartite tree $T\left(d_{A}, d_{B}\right)$ can be partitioned as $V=V_{1} U V_{2}$ where $\mathrm{p}_{1}=\mathrm{p}_{1}\left(\mathrm{~V}_{1}\right)$ and $\mathrm{p}_{2}=\mathrm{p}_{2}\left(\mathrm{~V}_{2}\right)$ are the corresponding average degrees of vertices in $\mathrm{V}_{1}$ and $\mathrm{V}_{2}$, respectively. It is easy to see that $\mathrm{p}_{1}=\mathrm{d}_{\mathrm{B}}$ and $\mathrm{p}_{2}=\left(\mathrm{d}_{\mathrm{A}}+\mathrm{d}_{\mathrm{B}}-1\right) / \mathrm{d}_{\mathrm{B}}$. This implies that $T\left(d_{A}, d_{B}\right)$ is a bipartite pseudo-regular graph. Consequently, its corresponding spectral radius is $\rho\left(T\left(d_{A}, d_{B}\right)=\sqrt{p_{1} p_{2}}=\sqrt{d_{A}+d_{B}-1}\right.$. Because $\mathrm{T}\left(\mathrm{d}_{\mathrm{A}}, \mathrm{d}_{\mathrm{B}}\right)$ is a $\mathrm{Z}_{2}$ graph, one obtains that

$$
\rho\left(T\left(d_{A}, d_{B}\right)\right)=\sqrt{M_{2}\left(T\left(d_{A}, d_{B}\right)\right) / m\left(T\left(d_{A}, d_{B}\right)\right)}
$$

Proposition 3 Let $d_{A} \geq 2$ and $d_{B} \geq 2$ be positive integers for which $d_{A} \neq d_{B}$ holds. Then there exist exactly two non-isomorphic balanced BPS trees $T\left(d_{A}, d_{B}\right)$ and $T\left(d_{B}, d_{A}\right)$, for which the following relations are fulfilled:

$$
\begin{array}{cl}
\text { i. } & \mathrm{m}\left(\mathrm{T}\left(\mathrm{d}_{\mathrm{A}}, \mathrm{d}_{\mathrm{B}}\right)\right)=\mathrm{m}\left(\mathrm{T}\left(\mathrm{d}_{\mathrm{B}}, \mathrm{d}_{\mathrm{A}}\right)\right)=\mathrm{d}_{\mathrm{A}} \mathrm{d}_{\mathrm{B}} \\
\text { ii. } & \mathrm{M}_{2}\left(\mathrm{~T}\left(\mathrm{~d}_{\mathrm{A}}, \mathrm{d}_{\mathrm{B}}\right)\right)=\mathrm{M}_{2}\left(\mathrm{~T}\left(\mathrm{~d}_{\mathrm{B}}, \mathrm{d}_{\mathrm{A}}\right)\right)=\mathrm{d}_{\mathrm{A}} \mathrm{d}_{\mathrm{B}}\left(\mathrm{d}_{\mathrm{A}}+\mathrm{d}_{\mathrm{B}}-1\right) \\
\text { iii. } & \rho\left(\mathrm{T}\left(\mathrm{d}_{\mathrm{A}}, \mathrm{d}_{\mathrm{B}}\right)\right)=\rho\left(\mathrm{T}\left(\mathrm{d}_{\mathrm{B}}, \mathrm{d}_{\mathrm{A}}\right)\right)=\sqrt{\mathrm{d}_{\mathrm{A}}+\mathrm{d}_{\mathrm{B}}-1 .} .
\end{array}
$$

Proof. Identity (i) is trivial. Considering the validity of Eq.(ii), it should be taken into consideration that $\mathrm{m}_{1, \mathrm{~d}_{\mathrm{B}}}=\mathrm{d}_{\mathrm{A}}\left(\mathrm{d}_{\mathrm{B}}-1\right)$ and $\mathrm{m}_{\mathrm{d}_{\mathrm{A}}, \mathrm{d}_{\mathrm{B}}}=\mathrm{d}_{\mathrm{A}}$. This implies that

$$
\mathrm{M}_{2}\left(\mathrm{~T}\left(\mathrm{~d}_{\mathrm{A}}, \mathrm{d}_{\mathrm{B}}\right)\right)=\mathrm{d}_{\mathrm{A}}\left(\mathrm{d}_{\mathrm{B}}-1\right) \mathrm{d}_{\mathrm{B}}+\mathrm{d}_{\mathrm{B}} \mathrm{d}_{\mathrm{A}}^{2}=\mathrm{m}\left(\mathrm{d}_{\mathrm{A}}+\mathrm{d}_{\mathrm{B}}-1\right)=\mathrm{M}_{2}\left(\mathrm{~T}\left(\mathrm{~d}_{\mathrm{B}}, \mathrm{d}_{\mathrm{A}}\right)\right)
$$


and $\sqrt{\frac{M_{2}\left(T\left(d_{A}, d_{B}\right)\right)}{m}}=\sqrt{\frac{M_{2}\left(T\left(d_{B}, d_{A}\right)\right)}{m}}=\sqrt{\frac{m\left(d_{A}+d_{B}-1\right)}{m}}=\sqrt{d_{A}+d_{B}-1}$

Because tree $T\left(d_{A}, d_{B}\right)$ is a BPS graph, the validity of Eq.(iii) follows directly from the Lemma 1.

If $\mathrm{d}_{\mathrm{A}} \neq \mathrm{d}_{\mathrm{B}}$ holds then the corresponding pair of strongly balanced trees $\mathrm{T}\left(\mathrm{d}_{\mathrm{A}}, \mathrm{d}_{\mathrm{B}}\right)$ and $\mathrm{T}\left(\mathrm{d}_{\mathrm{B}}, \mathrm{d}_{\mathrm{A}}\right)$ are called strongly balanced twin-like trees.

Remark 1 It can be easily shown that the first Zagreb index of strongly balanced trees can be computed by the following formula:

$$
\mathrm{M}_{1}\left(\mathrm{~T}\left(\mathrm{~d}_{\mathrm{A}}, \mathrm{d}_{\mathrm{B}}\right)\right)=\mathrm{d}_{\mathrm{A}}^{2}+\mathrm{d}_{\mathrm{A}} \mathrm{d}_{\mathrm{B}}^{2}+\mathrm{d}_{\mathrm{A}}\left(\mathrm{d}_{\mathrm{B}}-1\right)=\mathrm{d}_{\mathrm{A}}\left(\mathrm{d}_{\mathrm{A}}-1\right)+\mathrm{m}\left(\mathrm{d}_{\mathrm{B}}+1\right)
$$

Remark 2 The existence of an m-edge strongly balanced tree depends on the value of $\mathrm{m}$. For example, there is no 7-edge tree belonging to the family of strongly balanced trees.

Proposition 4 Let $\mathrm{m}$ be a positive integer. A strongly balanced tree $\mathrm{T}$ with $\mathrm{m}(\mathrm{T}) \geq$ 4 edge number exists if $m$ is not a prime number.

Proof. i) Let $m \geq 4$ be an even integer. Then there exist non-isomorphic strongly balanced trees $\mathrm{T}(2, \mathrm{~m} / 2)$ and/or $\mathrm{T}(\mathrm{m} / 2,2)$ with an identical spectral radius and a second Zagreb index. ii) Let $\mathrm{m} \geq 9$ be an odd integer which is not a prime number. In this case there exists at least one odd divisor $\mathrm{q}$ of $\mathrm{m}$. Consequently, $\mathrm{T}(\mathrm{q}, \mathrm{m} / \mathrm{q})$ will be a strongly balanced tree.

Corollary 1 Let $m \geq 6$ be an even integer. Then there exist at least two nonisomorphic strongly balanced m-edge trees $T\left(d_{A}, d_{B}\right)$ and $T\left(d_{B}, d_{A}\right)$ having an equal spectral radius and a second Zagreb index.

Example 4 The smallest strongly balanced tree graph pair of such type are the 7vertex $\mathrm{T}(3,2)$ and $\mathrm{T}(2,3)$ trees. It is important to note that 7 -vertex graphs $\mathrm{T}_{7, \mathrm{X}}$ and $\mathrm{T}_{7, \mathrm{Y}}$ depicted in Fig. 10 are isomorphic to trees $\mathrm{T}(3,2)$ and $\mathrm{T}(2,3)$ with $\rho(\mathrm{T}(3,2))=$ $\rho(\mathrm{T}(2,3))=2$.

The spectra of these strongly balanced trees are: $\operatorname{Spec}(\mathrm{T}(3,2))=\{2,1,1,0,-1,-1$ $2\}$ and $\operatorname{Spec}(\mathrm{T}(2,3))=\{2,1.4142,0,0,0,-1.4142,-2\}$. As can be observed, $\mathrm{T}(3,2)$ is a harmonic integral graph, but tree $\mathrm{T}(2,3)$ is a non-integral graph.

It is easy to construct a finite set of strongly balanced tree graphs having the same spectral radius. The method used for constructing such trees is demonstrated in the following example.

Example 5 Let $d_{A}+d_{B}=50$. It is easy to see that the strongly balanced trees denoted by $\mathrm{T}(2,48), \mathrm{T}(3,47), \ldots, \mathrm{T}(25,25), \ldots, \mathrm{T}(47,3)$ and $\mathrm{T}(48,2)$ have the same integer spectral radius $\rho=\sqrt{50-1}=7$. As an example, trees $\mathrm{T}(10,40)$ and $\mathrm{T}(40,10)$ are depicted in Fig. 14. 


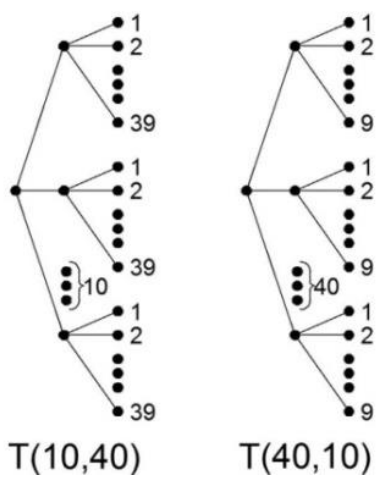

Figure 14

Twin-like trees TS(10,40) and TS(40,10) with 400 edges

Some computed results concerning Example 5 are summarized in Table 3.

Table 3

Computed topological parameters for strongly balanced trees

\begin{tabular}{|c|c|c|c|c|c|c|c|}
\hline Graph & $\mathbf{d}_{\mathbf{A}}$ & $\mathbf{d}_{\mathbf{B}}$ & $\boldsymbol{\rho}$ & ${\mathbf{m}=\mathbf{d}_{\mathbf{A}} \mathbf{d}_{\mathbf{B}}}$ & $\mathbf{M}_{\mathbf{2}}=\mathbf{m} \boldsymbol{\rho}^{\mathbf{2}}$ & $\begin{array}{c}\mathbf{M}_{\mathbf{1}}=\mathbf{d}_{\mathbf{A}}\left(\mathbf{d}_{\mathbf{A}^{-}}\right. \\
\mathbf{1} \mathbf{+} \mathbf{( \mathbf { d } _ { \mathbf { B } } + \mathbf { 1 } )}\end{array}$ & $\begin{array}{c}\mathbf{Z}=\mathbf{M}_{\mathbf{2}} / \mathbf{m}- \\
\mathbf{M}_{\mathbf{1}} \mathbf{n}\end{array}$ \\
\hline $\mathrm{T}(2,48)$ & 2 & 48 & 7 & 96 & 4704 & 4706 & 0.4845 \\
\hline $\mathrm{T}(3,47)$ & 3 & 47 & 7 & 141 & 6909 & 6774 & 1.2958 \\
\hline $\mathrm{T}(10,40)$ & 10 & 40 & 7 & 400 & 19600 & 16490 & 7.8778 \\
\hline $\mathrm{T}(20,30)$ & 20 & 30 & 7 & 600 & 29400 & 18980 & 18.3607 \\
\hline $\mathrm{T}(24,26)$ & 24 & 26 & 7 & 624 & 30576 & 17400 & 21.1600 \\
\hline $\mathrm{T}(25,25)$ & 25 & 25 & 7 & 625 & 30625 & 16850 & 22.0831 \\
\hline $\mathrm{T}(26,24)$ & 26 & 24 & 7 & 624 & 30576 & 16250 & 26.0000 \\
\hline $\mathrm{T}(30,20)$ & 30 & 20 & 7 & 600 & 29400 & 13470 & 34.1372 \\
\hline $\mathrm{T}(40,10)$ & 40 & 10 & 7 & 400 & 19600 & 5960 & 29.8028 \\
\hline $\mathrm{T}(47,3)$ & 47 & 3 & 7 & 141 & 6909 & 2726 & 22.7732 \\
\hline $\mathrm{T}(48,2)$ & 48 & 2 & 7 & 96 & 4704 & 2544 & \\
\hline
\end{tabular}

Based on the considerations outlined previously the following conclusions can be drawn:

i. The Bethe trees of 3 levels denoted by $\mathrm{B}_{\Delta, 3}$ and the completely fulldegree trees of 3 levels denoted by $\mathrm{T}_{\Delta, 3}$ are strongly balanced trees.

ii. Let $\mathrm{C} \geq 4$ be a positive integer. For strongly balanced trees $\mathrm{T}(2, \mathrm{C}-2)$ the following equality holds: $\mathrm{M}_{2}(\mathrm{~T}(2, \mathrm{C}-2))-\mathrm{M}_{1}(\mathrm{~T}(2, \mathrm{C}-2))=-2$.

iii. Let $\mathrm{C}_{1} \geq 4$ be an even positive integer. Among the strongly balanced trees the second Zagreb index has a maximum value if $\mathrm{d}_{\mathrm{A}}=\mathrm{d}_{\mathrm{B}}=\mathrm{C}_{1} / 2$ holds. This maximum value belongs to the strongly balanced tree $T\left(C_{1} / 2, C_{1} / 2\right)$. 
iv. Let $\mathrm{C}_{2} \geq 5$ be an odd positive integer. Among the strongly balanced trees, there exist two non-isomorphic trees having an identical maximum second Zagreb index. These trees are: $\left.\mathrm{T}\left(\left(\mathrm{C}_{2}-1\right) / 2,\left(\mathrm{C}_{2}+1\right) / 2\right)\right)$ and $\left.\mathrm{T}\left(\left(\mathrm{C}_{2}+1\right) / 2,\left(\mathrm{C}_{2}-1\right) / 2\right)\right)$.

v. If $d_{A}+d_{B}-1$ is a perfect square, then for trees $\left.T\left(d_{A}, d_{B}\right)\right)$ and $\left.T\left(d_{B}, d_{A}\right)\right)$ the corresponding spectral radius $\rho\left(T\left(d_{A}, d_{B}\right)\right)=\rho\left(T\left(d_{B}, d_{A}\right)\right)$ will be a positive integer.

Example 6 If $d_{A}=d_{B}=5$, then $d_{A}+d_{B}-1=9$ is a perfect square, consequently, the strongly balanced tree $\mathrm{T}(5,5)$ has an integer spectral radius $\rho(T(5,5))=\sqrt{5+5-1)}=3$. The completely full-degree tree $T_{5,3}$ depicted in Fig.11. is isomorphic to the strongly balanced tree $\mathrm{T}(5,5)$ which is an integral graph [22] with the spectrum $\operatorname{Spec}(T(5,5))=\left\{3,2^{4}, 0^{16},-2^{4},-3\right\}$. It is worth noting that among strongly balanced trees there are several integral graphs. For example, the smallest one is the 7-vertex tree $\mathrm{T}_{7, \mathrm{X}}=\mathrm{T}(3,2)$ shown in Fig. 10.

Proposition 5 There exist infinitely many strongly balanced trees which are extremal graphs having a minimal spectral radius.

Proof. Belardo et al. [40, 18] considered the family of n-vertex trees with diameter 4. Among these trees, they identified those trees whose spectral radius is minimal. It has been proved [40] that for any $\mathrm{n} \geq 5$, the minimum spectral radius among trees $T(n, 4)$ is attained only in two cases a) by a single tree $M_{n, 4}^{j}$ if $n \neq j^{2}+j+1$, and b) by tree pairs $\mathrm{MT}_{\mathrm{n}, 4}^{\mathrm{j}}$ and $\mathrm{MT}_{\mathrm{n}, 4}^{\mathrm{j}+1}$ if $\mathrm{n}=\mathrm{j}^{2}+\mathrm{j}+1$ for arbitrary $\mathrm{j} \geq 2$ integer.

It is easy to see that for even $m=n-1=j^{2}+j \quad(j=2,3,4, .$.$) the corresponding$ extremal m-edge trees $\mathrm{MT}_{\mathrm{n}, 4}^{\mathrm{j}}$ and $\mathrm{MT}_{\mathrm{n}, 4}^{\mathrm{j}+1}$ are strongly balanced tree graphs having an identical spectral radius. For extremal tree pairs $\mathrm{MT}_{\mathrm{n}, 4}^{\mathrm{j}}$ and $\mathrm{MT}_{\mathrm{n}, 4}^{\mathrm{j}+1}$ the possible vertex numbers are $n=7,13,21,31, \ldots$ This implies that among 7 -vertex trees of diameter 4 the strongly balanced trees $\mathrm{T}_{7, \mathrm{X}}=\mathrm{T}(3,2)$ and $\mathrm{T}_{7, \mathrm{Y}}=\mathrm{T}(2,3)$ depicted in Fig. 10 are extremal trees with identical minimal spectral radius $\rho\left(T_{7, X}\right)=\rho\left(T_{7, Y}\right)=\sqrt{3+2-1)}=2$

Proposition 6 There exist infinitely many strongly balanced trees E(p) which are harmonic and pseudo-semiregular simultaneously and they have integer spectral radii.

Proof. Starting with the concept outlined in [19], consider the infinite sequence of balanced tree graphs $E(p)=T\left(d_{A}(p), d_{B}(p)\right)$ constructed as follows: If $p \geq 2$, then trees $E(p)$ can be obtained from $d_{A}(p)=p^{2}-p+1$ disjoint stars $K_{1, p-1}$ by adding a vertex adjacent to the central vertex of each stars. It follows that the degree of central vertex is $d_{A}(p)=p^{2}-p+1$ and $d_{B}(p)=p$. Moreover, the corresponding edge number is $\mathrm{m}(\mathrm{E}(\mathrm{p}))=\left(\mathrm{p}^{2}-\mathrm{p}+1\right) \mathrm{p}$. 
Trees $\mathrm{E}(\mathrm{p})$ are harmonic, pseudo-semiregular and strongly balanced simultaneously, their spectral radii is

$$
\rho(E(p))=\sqrt{d_{A}(p)+d_{B}(p)-1}=\sqrt{p^{2}}=p
$$

If $\mathrm{p}=2$, tree $\mathrm{E}(2)$ is isomorphic to the 7-vertex graph $\mathrm{T}_{7, \mathrm{X}}$ depicted in Fig. 10. If $\mathrm{p}=3$, as a particular case, tree $\mathrm{E}(3)$ is isomorphic to the 22-vertex tree graph $\mathrm{G}_{\mathrm{D}}$ shown in Fig. 1.

Proposition 7 There exist infinitely many strongly balanced trees $F(p)$ which are non-harmonic, pseudo-semiregular graphs having integer spectral radii.

Proof. The construction of balanced trees $F(p)$ is based on the same concept presented previously in Proposition 6. Consider now the infinite sequence of strongly balanced trees defined as $F(p)=T\left(d_{B}(p), d_{A}(p)\right)$. Because $E(p)$ and $F(p)$ are strongly balanced twin-like trees, this implies that they have similar properties: their spectral radii $\rho(E(p))=\rho(F(p))=p$ are positive integers. The only significant difference between them is that trees $F(p)$ are not harmonic. If $p=2$, tree $F(2)$ is isomorphic to the 7-vertex graph $\mathrm{T}_{7, \mathrm{Y}}$ depicted in Fig. 10.

It is an interesting observation that there exists a broad class of connected graphs with maximum degree $\Delta=\Delta(\mathrm{G})$ for which the equality $\rho(G)=\sqrt{\Delta+\mathrm{p}}$ holds where $\mathrm{p}$ is a non-negative integer. These graphs can be classified into 3 disjoint subsets by considering the following relationships: $\mathrm{p}<\Delta, \mathrm{p}=\Delta$ and $\mathrm{p}>\Delta$.

For parameter $\mathrm{p}$ a simple upper bound can be obtained. Because for the spectral radius of a graph $\mathrm{G}$ the inequality $\rho^{2}(\mathrm{G}) \leq \Delta^{2}$ holds, this implies that $\Delta+\mathrm{p}=\rho^{2}(\mathrm{G})$ $\leq \Delta^{2}$ is fulfilled if $\mathrm{p} \leq \Delta(\Delta-1)$. The above inequality is sharp. Equality holds if $\mathrm{G}_{\mathrm{R}}$ is an R-regular graph. Because $\rho\left(G_{R}\right)=R=\Delta$, it follows that $\rho\left(G_{R}\right)=\sqrt{\Delta+p}$ is fulfilled if $\mathrm{p}=\mathrm{R}(\mathrm{R}-1)$.

It is easy to check that equality $\rho(G)=\sqrt{\Delta+p}$ holds for any $Z_{2}$ graph, because $\mathrm{p}=\mathrm{M}_{2} / \mathrm{m}-\Delta$ is a non-negative integer [16]. For example, equality with $\mathrm{p}=0$ is valid for all $\mathrm{n}$-vertex stars $\mathrm{K}_{1, \mathrm{n}-1}$.

Proposition 8 Let $\mathrm{p} \geq 1$ be an arbitrary positive integer. Then for any parameter $\mathrm{p}$ there exists a strongly balanced tree $K(p)$ for which $\rho(K(p))=\sqrt{\Delta+p}$ is fulfilled.

Proof. The construction of such trees is based on the following considerations. Starting with graphs $E(p)$, define the trees $K(p)$ as follows: $K(p)=E(p+1)$ for any positive integer $p$. Since $E(p)$ is harmonic and strongly balanced, one obtains on the one hand

$$
\begin{aligned}
& \rho(K(p))=\rho(E(p+1))=\sqrt{d_{A}(p+1)+d_{B}(p+1)-1}= \\
& \sqrt{\left((p+1)^{2}-(p+1)+1\right)+(p+1)-1}=\sqrt{(p+1)^{2}}=p+1
\end{aligned}
$$


On the other hand, because $\Delta(\mathrm{K}(\mathrm{p}))=\Delta(\mathrm{E}(\mathrm{p}+1))=\mathrm{d}_{\mathrm{A}}(\mathrm{p}+1)=(\mathrm{p}+1)^{2}-(\mathrm{p}+1)+1$ holds, we get

$$
\sqrt{\Delta(\mathrm{K}(\mathrm{p}))+\mathrm{p}}=\sqrt{\Delta(\mathrm{E}(\mathrm{p}+1))+\mathrm{p}}=\sqrt{\left((\mathrm{p}+1)^{2}-(\mathrm{p}+1)+1\right)+\mathrm{p}}=\mathrm{p}+1 .
$$

\section{Acknowledgement}

We acknowledge the financial support of this work by the Mexican-Hungarian S\&T bilateral project TÉT MX -1-2013-0001 and "Cooperative research on control of heat treatment stress and distortion for high value added machinery products" (2014DFG72020) projects.

\section{References}

[1] N. Biggs, Algebraic Graph Theory, Cambridge Univ. Press, Cambridge, 1974

[2] A. Yu, M. Lu, F. Tian, On the Spectral Radius of Graphs, Lin. Algebra Appl. 387 (2004) 41-49

[3] B. Borovićanin, S. Grünewald, I. Gutman, M. Petrović, Harmonic Graphs with a Small Number of Cycles, Discrete Math. 265 (2003) 31-44

[4] M. Petrović, B. Borovićanin, Z. Radosavljević, The Integral 3-Harmonic Graphs, Lin. Algebra Appl. 416 (2006) 298-312

[5] Y. Hou, Z. Teng, C. Woo, On the Spectral Radius, k-degree and the Upper Bound of Energy in a Graphs, MATCH Commun. Math. Comput. Chem. 57 (2007) 341-350

[6] P. Hik, R. Nadela, Balanced Integral Trees, Math. Slovaca 48 (1998) 429445

[7] S. Nikolić, G. Kovačević, A. Miličević, N. Trinajstić, The Zagreb Indices 30 Years After, Croat. Chem. Acta, 76 (2003) 113-124

[8] I. Gutman, K. Ch. Das, The First Zagreb Index 30 Years after, MATCH Commun. Math. Comput. Chem. 50 (2004) 83-92

[9] B. Furtula, I. Gutman, S. Ediz, On difference of Zagreb indices, Discr. Appl. Math. 178 (2014) 83-88

[10] B. Zhou, Zagreb indices, MATCH Commun. Math. Comput. Chem. 52 (2004) 113-118

[11] D. Vukičević, I. Gutman, B. Furtula, V. Andova, D. Dimitrov, Some observations on comparing Zagreb indices, MATCH Commun. Math. Comput. Chem. 66 (2011) 627-645

[12] I. Gutman, Degree-based Topological Indices, Croat. Chem. Acta 86 (2013) 351-361 
[13] B. Liu, Z. You, A Survey on Comparing Zagreb Indices, MATCH Commun. Math. Comput. Chem. 65 (2011) 581-593

[14] H. Abdo, D. Dimitrov, I. Gutman, On the Zagreb Indices Equality, Discr. Appl. Math. 160 (2012) 1-8

[15] A. Ilić, D. Stevanović, On Comparing Zagreb Indices, MATCH Commun. Math. Comput. Chem. 62 (2009) 681-687

[16] H. Abdo, D. Dimitrov, T. Reti, D. Stevanović, Estimation of the Spectral Radius of a Graph by the Second Zagreb Index, MATCH Commun. Math. Comput. Chem. 72 (2014) 741-751

[17] C. Elphick, T. Réti, On the Relations between the Zagreb Indices, Clique Numbers and Walks in Graphs, MATCH Commun. Math. Comput. Chem. 74 (2015) 19-34

[18] D. Stevanović, Spectral Radius of Graphs, Academic Press, Amsterdam, 2015

[19] P. Rowlinson, The Main Eigenvalues of a Graph: A Survey, Applicable Analysis and Discrete Mathematics, 1 (2007) 445-471

[20] E. R. van Dam, R. E. Krooij, The Minimal Spectral Radius of Graphs with a Given Diameter, Lin. Algebra Appl. 423 (2007) 408-419

[21] F. Harary, A.J. Schwenk, Which Graphs have Integral Spectra? In: R. Bari, F. Harary (Eds.), Graph and Combinatorics. Lecture Notes in Mathematics, 406, pp. 45-51. Springer, Berlin (1974)

[22] M. Watanabe, A.J. Schwenk, Integral Starlike Trees, J. Austral. Math. Soc. (Series A) 28 (1979) 120-128

[23] K. Balińska, D. Cvetković, Z. Radosavljević, S. Simić, D. Stevanović, A Survey on Integral Graphs, Univ. Beograd. Publ. Elektrotehn. Fak. Ser. Mat. 13 (2002) 42-65

[24] Z. Tang, Y. Hou, Integral Graphs with Index 3 and Exactly Two Main Eigenvalues, Lin. Algebra Appl. 433 (2010) 984-993

[25] Ligong Wang, Integral Trees and Integral Graphs, Ph.D. Thesis, University of Twente, 2005

[26] A. E. Brouwer, W. H. Haemers, The Integral Trees with Spectral Radius 3, Lin. Algebra Appl. 429 (2008) 2710-2718

[27] P. Hik, M. Pokorny, There are Integral Tees of Diameter 7, Univ. Beograd. Publ. Elektrotehn. Fak. Ser. Mat. 18 (2006) 59-63

[28] P. Csikvári, Integral Trees of Arbitrary Large Diameters, J. Algebr. Combin. 32 (2010) 371-377

[29] E. Ghorbani, A. Mohammadian, B.Tayfeh-Rezaie, Integral Trees of Odd Diameters, J. Graph Theory, 70 (2011) 332-338 
[30] L. Patuzzi, M. A. A. de Freitas, R.R. Del Vecchio, Integer Index in Trees of Diameter 4, Filomat, 28:2 (2014) 241-248

[31] L. Patuzzi, M. A. A. de Freitas, R.R. Del Vecchio, Indices for Special Classes of Trees, Lin. Algebra Appl. 442 (2014) 106-114

[32] E. R. van Dam, Graphs with given Diameter Maximizing the Spectral Radius, Lin. Algebra Appl. 426 (2007) 454-457

[33] P. Hansen, D. Stevanović, On Bags and Bugs, Discrete Appl. Math. 156 (2008) 986-997

[34] X. Y. Yuan, J. Y. Shao, Y. Liu, The Minimal Spectral Radius of Graphs of Order n with Diameter n - 4, Lin. Algebra Appl. 428 (2008) 2840-2851

[35] L. Lovász, J. Pelikán, On the Eigenvalues of Trees, Periodica, Math. Hung. 3 (1973) 175-182

[36] S. Simić, D. V. Tošić, The Index of Trees with Specified Maximum Degree, MATCH Commun. Math. Comput. Chem. 54 (2005) 351-362

[37] D. Stevanović, Bounding the Largest Eigenvalue of Trees in Terms of the Largest Vertex Degree, Lin. Algebra Appl. 360 (2003) 35-42

[38] H. Song, Q. Wang, L. Tian, New Upper Bounds on the Spectral Radius of Trees with the Given Number of Vertices and Maximum Degree, Lin. Algebra Appl. 439 (2013) 2527-2541

[39] A. Chang, Q. Huang, Ordering Trees by their Largest Eigenvalues, Lin. Algebra Appl. 370 (2003) 174-184

[40] F. Belardo, EM. Li Marzi, S. Simić, Trees with Minimal Index and Diameter at most Four, Discrete Math. 310 (2010) 1708-1714

[41] O. Rojo, M. Robinho, An Explicit Formula for Eigenvalues of Bethe Trees and Upper Bounds on the Largest Eigenvalue of any Tree, Lin. Algebra Appl. 427 (2007) 138-150

[42] B. Wu, E. Xiao, Y. Hong, The Spectral Radius of Trees on k Pendant Vertices, Lin. Algebra Appl. 395 (2005) 343-349

[43] J. H. Smith, Some Properties of the Spectrum of a Graph, in: R. Guy, H. Hanani, N. Sauer, J. Schönheim (Eds.) Combinatorial Structures and Their Applications, Gordon and Breach, Science Publ. Inc., New York, London, Paris, 1970, 403-406 